\title{
Damage evolution in marble under uniaxial compression monitored by Pressure Stimulated Currents and Acoustic Emissions
}

\author{
Ilias Stavrakas \\ Electronic Devices and Materials Laboratory, University of West Attica, Greece \\ ilias@uniwa.gr, bttp://orcid.org/0000-0001-8484-8751
}

Stavros K. Kourkoulis

National Technical University of Athens, Department of Mechanics, Athens, Greece

stakkoun@central.ntua.gr, bttps://orcid.org/0000-0003-3246-9308

Dimos Triantis

Electronic Devices and Materials Laboratory, University of West Attica, Greece

triantis@uniwa.gr,bttp://orcid.org/0000-0003-4219-8687

\begin{abstract}
The spatiotemporal evolution of damage in marble specimens under uniaxial compression is monitored using Pressure Stimulated Currents (PSCs) and Acoustic Emissions (AEs). The novelty of the study is the use of an integrated grid of sensors (instead of a single pair of electrodes) to detect the weak electrical signals, emitted during loading. The use of such a grid of sensors does indeed enhance the capabilities of the PSC technique providing valuable information about the initiation and propagation of micro-fracturing at the interior of the specimens. The experimental results indicate that both the improved b-value of the AE hits and the energy of the PSCs offer information about the proximity of the applied stress to that causing fracture. It is thus concluded that both quantities could be considered as pre-failure indicators.
\end{abstract}

KEYWORDS. Pressure Stimulated Currents; Acoustic Emissions; Improved bvalue; Damage evolution; Uniaxial compression; Marble.

\section{OPEN ACCESS}

Citation: Stavrakas, I., Kourkoulis, S.K., Triantis, D., Damage evolution in marble under uniaxial compression monitored by Pressure Stimulated Currents and Acoustic Emissions, Frattura ed Integrità Strutturale, 50 (2019) 573-583.

Received: 16.01 .2019

Accepted: 15.05 .2019

Published: 01.10.2019

Copyright: (C) 2019 This is an open access article under the terms of the CC-BY 4.0, which permits unrestricted use, distribution, and reproduction in any medium, provided the original author and source are credited.

\section{INTRODUCTION}

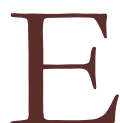
nlightening the spatiotemporal evolution of damage at the interior of structural elements under mechanical load is an interesting, though demanding, task of Mechanics of Materials and Engineering. Its importance becomes evident by taking into account that, in case damage evolution is properly monitored, the respective data could be used, among others, as tools for assessing the element's remaining life or, equivalently, as valuable pre-failure indicators. 
The quest for pre-failure indicators attracts long ago the interest of engineers and researchers from quite a few scientific fields, ranging from Civil Engineering to the Management of Natural Hazards and Natural Disasters. The key concept in studies dealing with pre-failure indicators is to understand the mechanisms leading to damage evolution within the volume of loaded structural elements. It is obvious that traditional strain/displacement sensing techniques used in Mechanics of Materials (like, for example, electric strain gauges, caustics, photoelasticity, Moiré fringes, interferometry etc.) are not suitable for such applications. Indeed, such techniques can only provide data from the external surface of the loaded members while it is definitely known that, well before any kind of damage is detected at the member's outer surface, internal damage mechanisms have been already activated at the member's interior, guiding in fact the phenomena observed on the member's surface. The same is true for some modern (and flexible for many other purposes) sensing techniques like, for example, Digital Image Correlation [1] and Direct Strain Imaging [2]. In this context, description of the mechanical response of materials is usually achieved with the aid of analytic and numerical models, based on experimental data concerning the macroscopic mechanical properties of the material (or of its constituent materials in case of composite structures).

In the direction of overcoming the above difficulties, various experimental techniques have been developed during the last decades, aiming to the quantitative description of the structural changes, which take place at the interior of mechanically loaded structures (both before and during the initiation and propagation of micro-fracturing processes). Among them, the Acoustic Emission (AE) technique (i.e., the detection and analysis of the characteristics of transient elastic waves caused by the rapid release of strain energy during mechanical loading when micro-fracture phenomena take place) is, perhaps, the most mature and widely used one. It is based on firm and well understood physical principles [3-7].

Recently, another promising experimental technique, permitting insight at the interior of loaded materials, has been introduced $[8,9]$, based on the detection of weak electrical current emissions, usually called Pressure Stimulated Currents (PSCs). These currents (the magnitude of which is of the order of $\mathrm{pA}$ ), produced during mechanical loading, are detected using extremely sensitive electrometers. This technique, known as PSC technique, has been successfully used for both natural building stones $[10,11]$ and artificial materials, like cement-based ones [12,13]. It is already adopted by many researchers [14-16] worldwide while other researchers employ techniques [17, 18] based on the same natural principles.

The two sensing techniques mentioned above (AE, PSC) are here employed in a combined manner in order to monitor damage evolution within Dionysos marble specimens subjected to uniaxial compression until fracture. The novelty of the study (besides the combined use of the two techniques and the comparative analysis of the respective data) is that the PSC technique is applied, for the first time, using a grid of sensors rather than a single pair of electrodes. The specific system permits gathering data from multiple electrical current channels. Valuable information is, thus, gained, concerning the spatial and the temporal distribution of micro-cracking processes, which precede final catastrophic crack propagation.

The combined analysis of the experimental results indicates that the specific arrangement of electrical sensors provides a clear overview of the spatiotemporal evolution of damage at the interior of the loaded specimen. Moreover, it is indicated that the time evolution of the improved b-value (I $b$-value) $[15,19]$ of the AE hits and the energy of the PSCs provide reliable indicators of of upcoming catastrophic failure.

\section{EXPERIMENTAL PROTOCOL}

\section{The material and the specimens}

The specimens of the present experimental protocol are made of Dionysos marble, an extremely fine-graded white marble quarried from Mount Dionysos in Attica region. It is extensively used, among others, by the scientific personnel of the restoration project of the Athenian Acropolis monuments for the construction of copies of lost or destroyed members. Dionysos marble is composed by about $98 \%$ of calcite, $0.5 \%$ of muscovite, $0.3 \%$ of sericite, $0.2 \%$ of quartz and $0.1 \%$ of chlorite [20]. It is emphasized here, that the extremely low quartz content is crucial for the present protocol, since it ensures that the electrical currents recorded are by no means related to the familiar piezoelectric effect. The specific and apparent densities of Dionysos marble are equal to about $2730 \mathrm{~kg} / \mathrm{m}^{3}$ and $2717 \mathrm{~kg} / \mathrm{m}^{3}$, respectively. The thermal expansion coefficient between $15{ }^{\circ} \mathrm{C}$ and $100{ }^{\circ} \mathrm{C}$ is $9 \times 10^{-6}{ }^{\circ} \mathrm{C}^{-1}$. The coefficient of absorption by weight is about $0.11 \%$. Its porosity is extremely low, ranging between $0.3 \%$ (virgin state) and $0.7 \%$ (superficial porosity) [20]. Its low porosity is another crucial aspect for the present study, since it blocks any electrokinetic effects (due to the existence of natural moisture) that would affect the behaviour of the electrical currents detected and recorded during the experiments.

From the Mechanics of Materials point of view, Dionysos marble is of orthotropic nature. Data for its mechanical properties, reported in literature, vary between very broad limits [21-24]. Based on a long series of experiments Vardoulakis et al. [21, 24] drew the conclusion that the mechanical properties along two of the anisotropy directions are close to each other and therefore the material is usually considered as transversely isotropic, described with the aid of only five elastic constants. 
In addition, these experiments indicated that Dionysos marble is slightly non-linear (both under tension and compression) and, also, slightly bimodular (the elastic modulus under compression exceeds that under tension by about $15 \%$ ).

The specimens prepared were prismatic of square cross section $40 \mathrm{x} 40 \mathrm{~mm}^{2}$. Their length was equal to $\mathrm{L}=100 \mathrm{~mm}$. Special attention was paid during the preparation of the specimens for their longitudinal axis to be normal to the material layers, in an attempt to avoid scattering of the experimental results due to the anisotropy of Dionysos marble.

\section{The experimental set-up}

The main experimental protocol consists of a series of uniaxial compression tests with a specially designed loading scheme. The specific loading model was chosen due to its increased practical importance for quasi-brittle rock and rock-like materials. An additional factor taken into account, in order to choose compression tests, is the fact that the orientation of the fracture planes (along which increased acoustic activity is expected after a load threshold) is more or less clearly predefined.

The force was applied normally to the material layers. The experiments were carried out using an INSTRON-DX 300 loading frame of capacity equal to $300 \mathrm{kN}$ under quasi-static loading conditions. Preliminary tests indicated that the compressive strength, $\sigma_{\mathrm{f}}$, of the specific block of Dionysos was equal to about $\sigma_{\mathrm{f}}=63.8 \mathrm{MPa}$.

Concerning the PSC technique, the sensing system consisted of an ultra-sensitive programmable electrometer (Keithley, $6517 \mathrm{~A}$ ) resolving currents ranging from $0.1 \mathrm{fA}$ to $20 \mathrm{~mA}$ in 11 ranges. The system was further supported by an electrical current 10-channel scanner card (Keithley 6521). The data of the electrometer were stored in a computer using a GPIB interface. The sensing system consisted of five pairs of electrodes, installed along the loading axis, enabling the collection of electric emissions as close as possible to any potential source of electric current (or, equivalently, to any point where damage occurs). The distance of the uppermost and the lowest pairs of electrodes from the upper and lower bases of the specimens, respectively, was $10 \mathrm{~mm}$ and the distance between two successive pairs of electrodes was $20 \mathrm{~mm}$ (Fig.1).

For the detection of acoustic emissions the 2-channel PCI-2 AE detection system was used (Physical Acoustics Corp). Two $\mathrm{R} 15 \alpha$ acoustic emission sensors $(150 \mathrm{kHz}$ resonant frequency) were placed at the positions shown in Fig. $1 \mathrm{a}$. The AE sensors were properly attached on the specimens by means of vacuum grease. Preamplifiers with $40 \mathrm{~dB}$ gain were used. The threshold of the AE amplitudes was set at $40 \mathrm{~dB}$.

In addition to the electrodes and the AE sensors, an electric strain gauge was glued at the center of one of the free lateral surfaces at the mid-height of the specimens, in order to measure the axial strain developed. Kyowa strain gauges were used, together with the Microlink 770 resistor bridge. Both the force exerted and the strain developed were digitized and stored to a computer using the KUSB-3100 (manufactured by Keithley) A/D data acquisition module.

Special attention was paid in all tests for the minimization of friction. The specific factor is of crucial importance, as it was pointed out by Labuz and Bridell [25]. In addition, Drescher and Vardoulakis [26] and Read and Hegemier [27] have definitely indicated that little can be inferred from non-lubricated tests. In this direction, two sheets of Polytetrafluoroethylene (PTFE) were interposed between each loading platen and the respective base of the specimen. In addition, steatic acid was spread between both the specimen's surface and the 'external' PTFE sheet and also between the two PTFE sheets. A similar

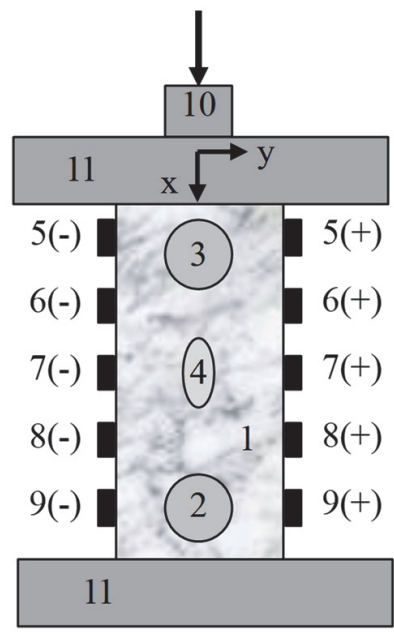

(a)



(b)

Figure 1: (a) Sketch of the experimental set-up (1: Specimen, 2,3: Lower and upper acoustic sensors, 4: Strain gauge, 5(+) to 9(+): Positive edge of the five successive channels (chan0-chan4) of the electrometer, 5(-) to $9(-)$ : Negative edge of the five successive channels, 10: Load cell, 11: Loading platens). (b) Photo of a typical specimen and the position of the sensors of the PSC technique. 
lubrication technique was used by van Vliet \& van Mier [28] with excellent results. It is mentioned here that the PTFE sheets provided also electrical insulation, a crucial parameter for the application of the PSC technique.

\section{The main experimental protocol and the loading scheme}

The specimens were subjected to sequential, load-control, loading-unloading-reloading loops, up to stress levels very closely approaching the compressive fracture strength of Dionysos marble. In fact, the stress level attained was equal to about $98 \%$ to $99 \%$ of the compressive strength, as it was determined during the preliminary tests. After the specific stress level (equal to about $62.7 \mathrm{MPa}$ ) was attained for the first time the externally applied compressive force was kept constant for a relatively long time interval equal to about $t_{\mathrm{L}}=150 \mathrm{~s}$. This step is considered necessary in order for the PSC to return to its initial background level and also for the activity recorded by the AE sensors to be eliminated. After this time interval the mechanical load was removed and the specimen remained unloaded for a time interval equal to about $t_{U}=50 \mathrm{~s}$. This procedure was repeated until the fracture of the specimen, which usually occurred during the second or the third loop. A typical loading protocol is shown in Fig.2, in which the axial stress is plotted versus time in juxtaposition to the respective axial strain. The specific specimen collapsed suddenly during the second loading loop in its constant force branch.



Figure 2: The time variation of the applied stress and the corresponding axial strain during the whole experimental procedure.

The respective axial stress - axial strain pairs, for the as above experiment, are plotted in Fig.3a. An almost perfectly linear region characterizes the first loading branch up to a stress level exceeding $50 \mathrm{MPa}$. The elastic modulus calculated from this branch is equal to about $\mathrm{E}_{1}=76.2 \mathrm{GPa}$, in very good agreement with results of similar previous experimental projects $[21,24]$. The non-reversible strain recorded after the load removal, equal to about $2 \times 10^{-4}$, is, also, in good agreement with the respective results from previously published works with Dionysos marble [29, 30]. As it is expected, during the loading branch of the second loading loop, the linear region of the stress-strain curve extends at higher stress levels (approaching $85 \%$ of the maximum compressive stress attained). In addition, the corresponding value of the elastic modulus becomes lower, equal to about $\mathrm{E}_{2}=61.8 \mathrm{GPa}$, again in good accordance with previously published data by Kourkoulis et al. [30], who have described the damage evolution in Dionysos marble in terms of the decrease of its elastic modulus versus the non-reversible portion of the strain, as recorded after successive loading-unloading-reloading loops. Taking advantage of their data, Fig. $3 \mathrm{~b}$ is plotted. In this figure the modulus of elasticity is plotted against the non-reversible part of the total strain, $\varepsilon_{\text {perm. }}$. On the same figure, the pair of $\varepsilon_{\text {perm }}$ and $E_{2}$ values obtained from Fig.3a (i.e., $\varepsilon_{\text {perm }}=2 \times 10^{-4}$ and $E_{2}=61.8 \mathrm{GPa}$ ) are indicated (see the red arrowed lines) clearly concluded that the damage level imposed during the first loading branch of the two-loops loading procedure approached very closely the terminal damage level that can be sustained by Dionysos marble. This close approach justifies the sudden collapse of the specific specimen during the second loading loop, even at its constant force branch.

Concerning the fracture mode, it is interesting to note that all specimens failed by almost axial splitting rather than along inclined planes, as it is suggested by the classical Mohr-Coulomb theory. In other words, in the specific series of tests the familiar Mohr's cones were almost totally suppressed. Although such behaviour is not common in standardized laboratory tests, it can be attributed to the excellent lubrication conditions achieved. The specific behaviour was long ago observed experimentally by Vardoulakis et al. [31] (Fig.3c). To explain this observation the so-called "post-peak stress diffusion" theory was introduced, that is capable of describing fracture of brittle materials by axial splitting rather than along inclined (with respect to the load axis) planes [31]. The theory is based on an alternative normalization approach: Instead of normalizing the axial stress over the area of the specimen's cross-section (which according to Van Vliet and van Mier [32] is inadequate for 


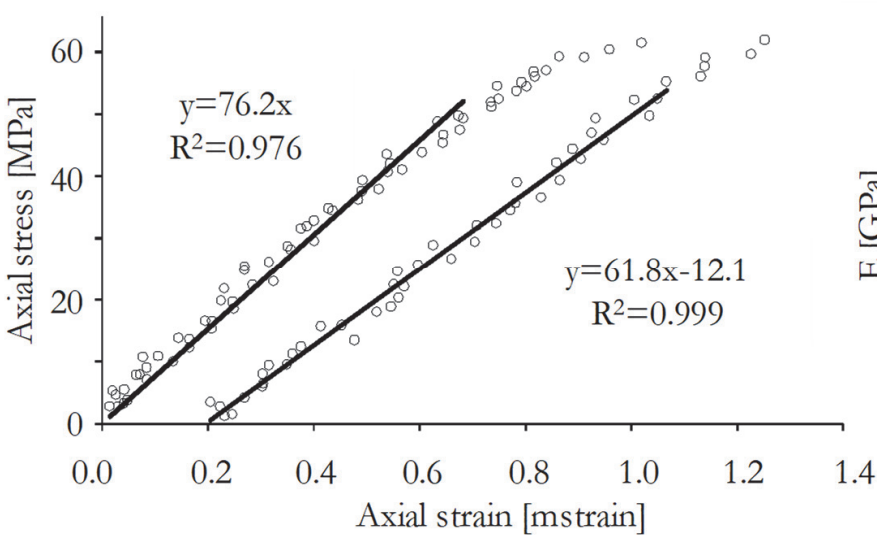

(a)



(b)

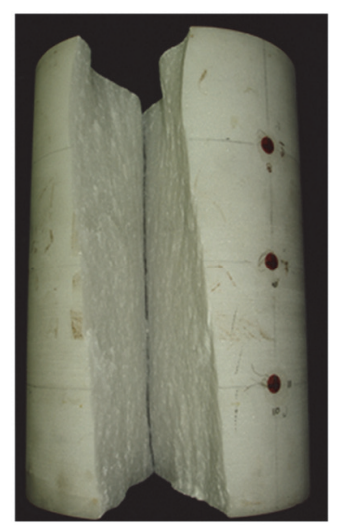

(c)

Figure 3: (a) The applied stress versus the corresponding axial strain during the two loading branches. (b) The degradation of the elastic modulus of Dionysos marble in terms of the non-reversible strain, $\varepsilon_{\text {perm }}$ [30]. (c) A specimen made of Dionysos marble loaded up to fracture under monotonic axial compression. The lubrication system used (combination of PTFE sheets and steatic acid) was suggested by Vardoulakis et al. [31]. It is clear that Mohr's cones are almost completely suppressed.

the description of the compression test beyond the peak stress), the axial stress is normalized over its peak value, and it is plotted versus the post-peak axial displacement. Describing the experimental results in this way provided very good correlations [32]. The main advantage of the "post-peak stress" diffusion theory is that it is totally independent of the strain concept, which is meaningless in the post-peak regime. In addition, it is quite advantageous that this theory permits description of the experimental results with a minimum number of parameters.

\section{EXPERIMENTAL RESULTS AND DISCUSSION}

\section{Spatiotemporal variation of the PSC}

$\mathrm{I}$ $\mathrm{n}$ previously published studies, in which the PSC technique was employed, a single pair of electrodes was used as sensing tool. In spite of this limitation, it was definitely concluded that, when marble specimens are subjected to compression, the electric activity within the volume of the specimens starts almost simultaneously with the damage processes. It is then gradually intensified with increasing stress level and reaches its maximum value when final fracture is impending [33]. After this maximization, a steep decrease of the PSC is systematically observed, designating exhaustion of the specimen's load carrying capacity, even though the specimen is not yet macroscopically fragmented.

In the present study the five pairs of electrodes attached on the specimens (Fig.1) permitted, in addition, a (more or less) accurate matching of the electrical signals detected to the locus at which they were generated. The main reason for using five pairs instead of a single one is based on the simple argument that an electrical signal that is generated within the mass of the specimen will reach an electrode of the electrometer attenuated, in accordance with the path that it followed, i.e., to the distance from the electrode. Assuming that the specimen is, more or less, homogeneous (and therefore it has the same electric resistance all over its volume) it can be concluded that when a damage event occurs in the vicinity of an electrode, the specific electrode will record higher electrical signal level while the electrodes at longer distances will also receive the signal but they will record it with lower amplitude. In this direction, all the channels of the electrometer are levelled and calibrated with a common reference in order to record electrical signals of the same source amplitude as equal.

The time variation of the axial stress and strain as well as the electrical signals received from each one of the five channels is shown in Fig.4, for a typical specimen that failed during the second loading loop (the specimen for which the loading scheme is shown in Fig.2). The experimental results indicated that during both loading branches (i.e., with increasing stress level) a systematic PSC increase is recorded from all the electrometer channels. When the applied stress reaches its maximum value, and it is then kept practically constant, the PSC emission follows a restoration process back to a background level, in agreement with previously published works [34, 35].

The fact that during the loading branch of the first loading loop the PSC emission is initially recorded stronger by channels 1, 2 and 3 (i.e., the ones located closer to the area around the mid-height of the specimen) indicates that for the specific specimen mechanical damage starts in the vicinity of its central cross section and it is gradually developed towards its bases. During the second loading loop, and while the mechanical stress was kept constant for about 100 s (i.e., at the time 
$1^{\text {st }}$ loading
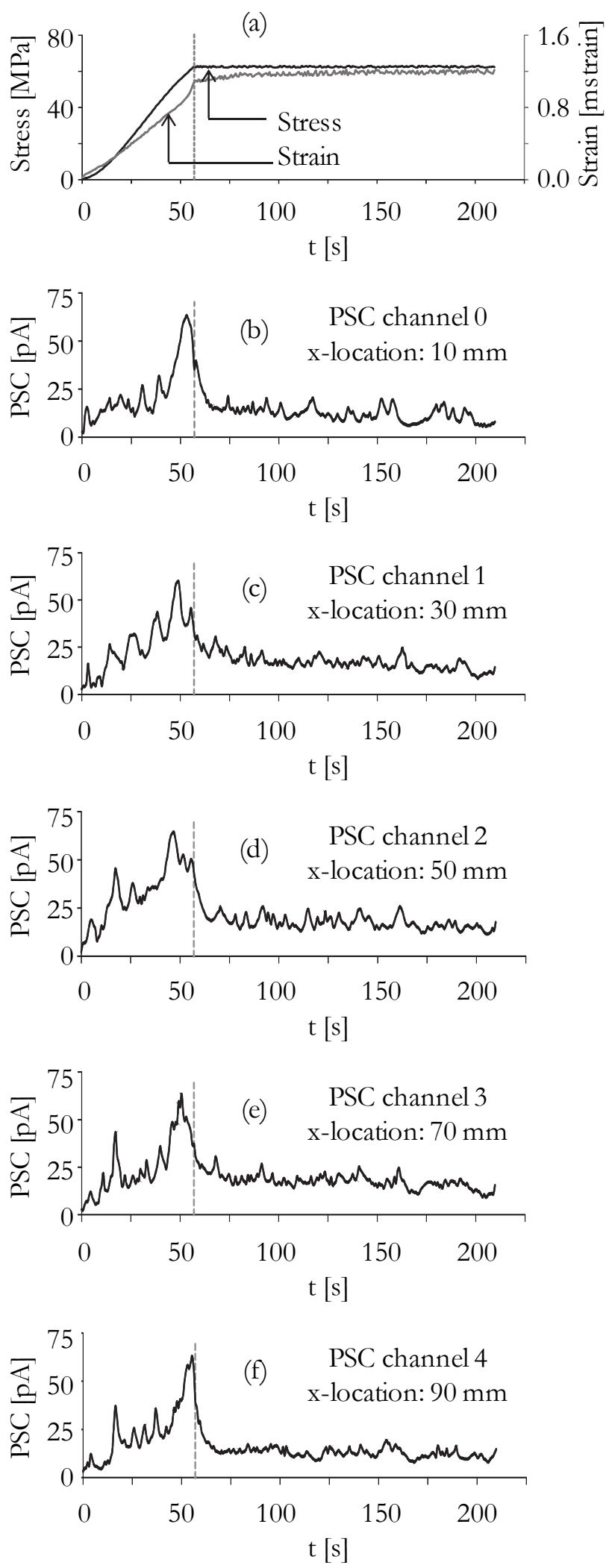

$2^{\text {nd }}$ loading
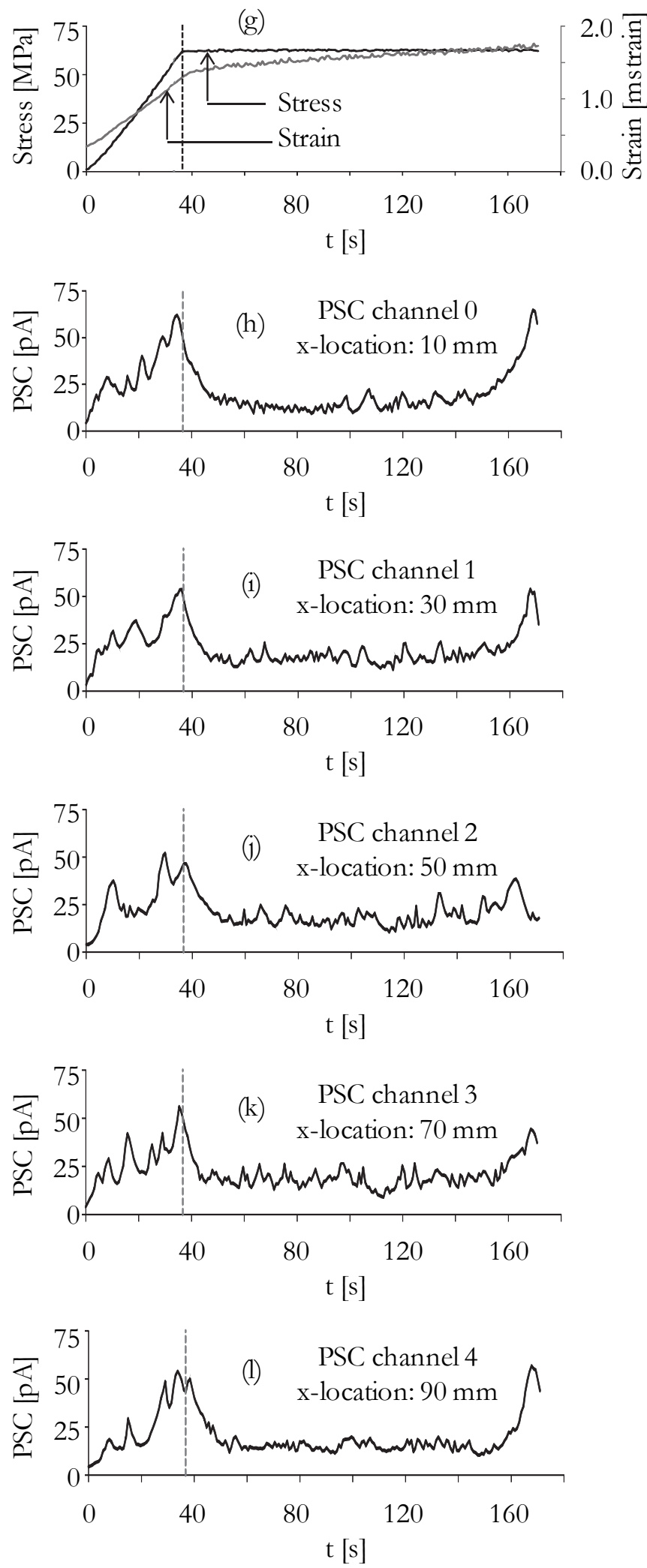

Figure 4: Time variation of the axial stress and the axial strain $(\mathrm{a}, \mathrm{g})$ and the corresponding behaviour of the electrical current emissions, as recorded by the PSC channels 0-4 (b-f, h-l), for the first (left column) and the second (right column) loading loops. 
instant with $\mathrm{t}=140 \mathrm{~s}$ in Fig.4) a gradual increase of the PSC was detected from all electrodes, designating the upcoming collapse of the specimen, which occurred about $30 \mathrm{~s}$ later, under constant external load. It is worth noting that, contrary to what happened during the loading branch of the first loop, the PSC increase was now stronger at the edge electrodes (i.e., channel-0 and channel-4). It is thus indicated, that catastrophic cracking started at points closer to the bases rather than at the mid-height area of the specimen (although at the initial loading steps damage was more intense around the specimen's mid-height).

\section{The time variation of the AE data}

The time variation of (i) the amplitudes of the AE hits recorded from both the AE sensors used (Fig.1a), and of (ii) the rate (per second) of the AE hits is shown in Figs.5(a,b), respectively, for the first loading loop, for the as above mentioned specimen. The corresponding plots for the second loading loop are shown in Figs.5(c,d). Comparing the behaviour of the amplitudes of the AEs recorded during the two loading loops, it is clearly seen that the loading branch of the second loop is accompanied by AEs of significantly lower amplitudes. This observation clearly supports the dominance of the Kaiser effect [36]. In addition, it becomes clear that, during the first loading loop and after the maximum stress level is attained, the AEs recorded are of lower amplitude. On the other hand, during the second loading cycle, and after reaching the maximum stress level, the number of AE hits increases and it is kept almost constant for about $100 \mathrm{~s}$ (Fig. 5d). From this point on, the number of AE hits rate starts increasing and their amplitudes become gradually higher until the final fracture of the specific specimen, which occurred at a time instant around $\mathrm{t}=170 \mathrm{~s}$. Recapitulating, the AE activity is much weaker during the loading branch of the second loading loop, as it is well established in literature [37-39].
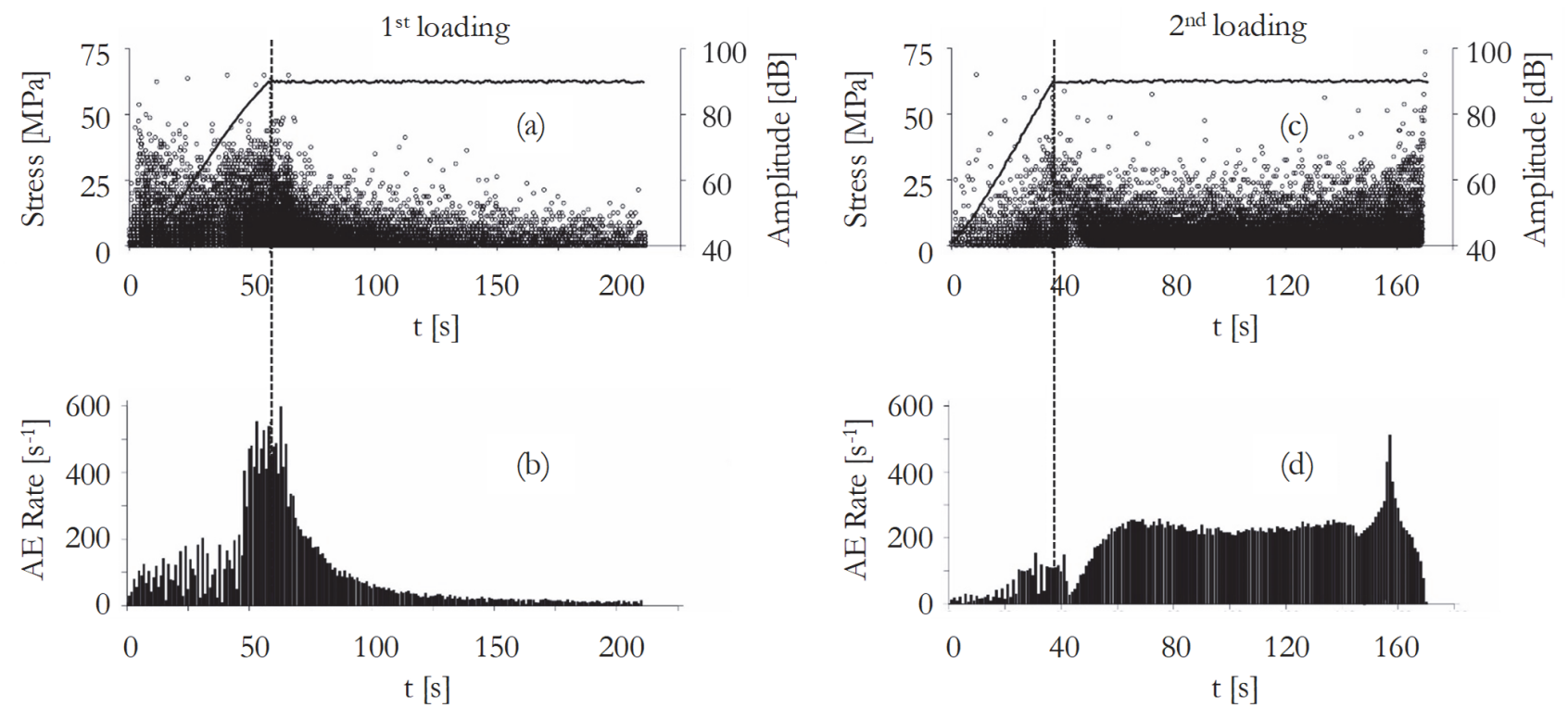

Figure 5: Time variation of $(\mathrm{a}, \mathrm{c})$ the axial stress and the corresponding amplitudes of the AE hits, and, (b, d) the corresponding behaviour of the rate of the $\mathrm{AE}$ hits $\left(\mathrm{s}^{-1}\right)$, for the first (left column) and the second (right column) loading loop.

Similar conclusions can be drawn for the time variation of the rate of the AE hits during the two loading loops, which is plotted in Figs.5(b,d). Indeed, during the loading branch of the first loop the rate of the AE hits is significantly higher than the respective rate during the second loop, reaching a maximum value of about $600 \mathrm{AE}$ hits/s. The corresponding value for the loading branch of the second loop is limited to only $200 \mathrm{AE}$ hits/s, demonstrating again the existence of the Kaiser effect. It is interesting to notice the sudden increase of the AE hits rate only a short while after the maximum stress value is attained during the second loading loop (although the stress level is kept constant). This is a clear sign that some kind of dynamic process is activated, which will soon lead to the final fracture of the specimen. The sudden increase of the rate of the AE hits, a little before the final fracture, is another pre-failure indicator that is worth to be highlighted.

\section{Correlation between PSC and AE data}

The correlation between the data regarding electric and acoustic emissions is here attempted in terms of the PSC energy and the corresponding time variation of the AE I $b$-value. More specifically, the PSC energy is calculated according to Eq.(1), after applying proper de-noise and background elimination filters on the signals recorded: 


$$
E(t) \propto \int_{t}^{t+\Delta t} P S C^{2}(t) d t
$$

In order to compare the PSC energy for each one of the electrode pairs and estimate the source location of the electrical emissions, as well as their time variation, Matlab scripts were prepared. The results of this procedure are presented (in juxtaposition to the respective time variation of the axial stress and axial strain, which are plotted again in Figs.6(a,d) for convenience) for both loading loops in Figs.6(b,e). The colour variation in these figures corresponds to the level of the PSC energy (according to the scale shown in the figure embedded between Figs.6b and 6e) while the y-axis represents the $\mathrm{x}$-location on the specimen.

From Fig.6b it is clearly seen that during the loading branch of the first loading loop and for stress values which do not exceed about $50 \%$ of the respective maximum value attained, electrical signals of relatively low energy are recorded. These signals may be well attributed to the pore closing process. Increasing further the stress level, high energy PSC emissions are recorded approximately at the mid-height of the specimen spreading gradually towards its bases. While the axial stress is then kept constant at its maximum level, the PSC energy is relaxed back to a background level although the strain recorded does not exhibit significant variations.

During the second loading loop the maximum values of the PSC energy were lower compared to the corresponding ones of the first loop (Fig.6e), as it could be perhaps expected according to the Kaiser effect [36]. It is to be noticed here that during this second loading loop, and while the stress is kept constant at its maximum level, the corresponding strain keeps increasing though at a relatively low rate. However, what is astonishingly interesting in Fig.6e is that, well before the final fracture of the

$1^{\text {st }}$ loading
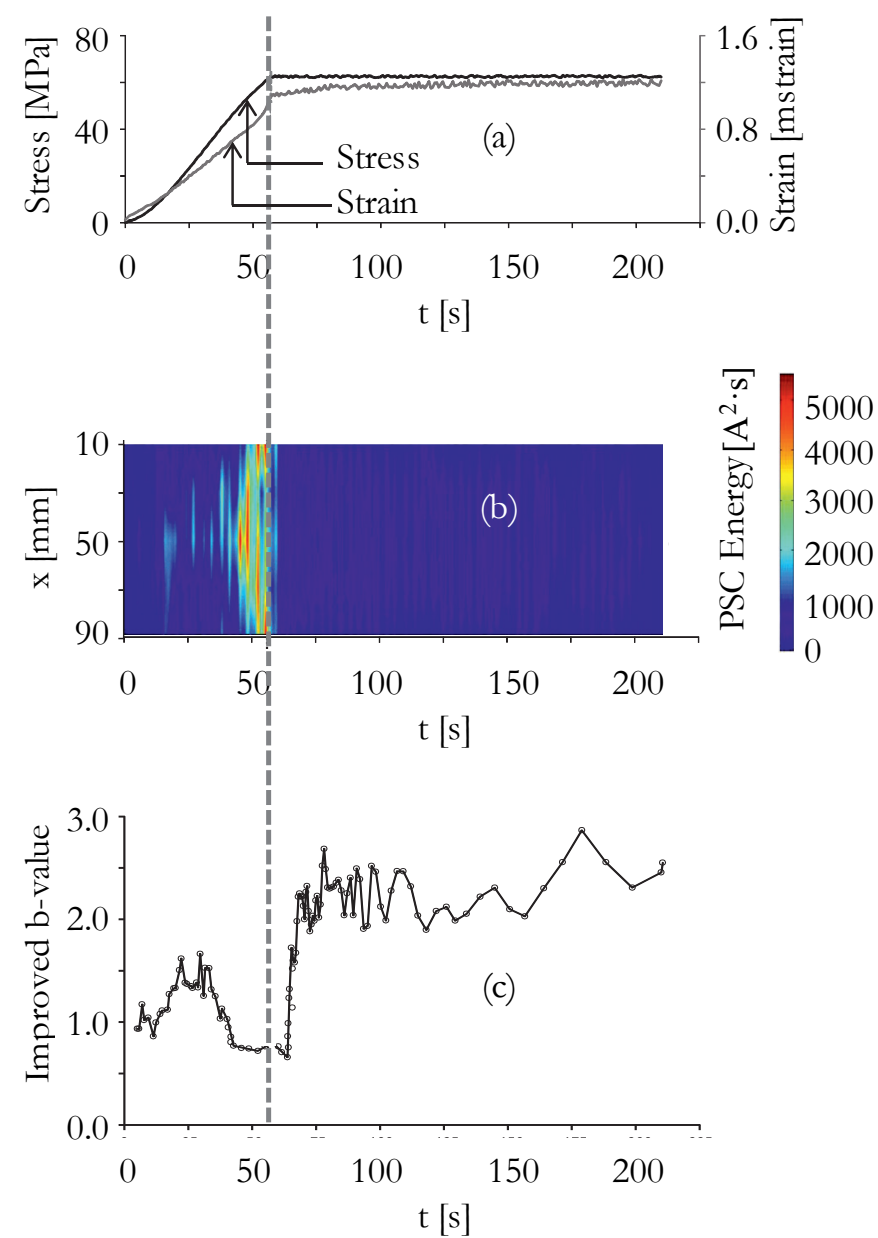

$2^{\text {nd }}$ loading
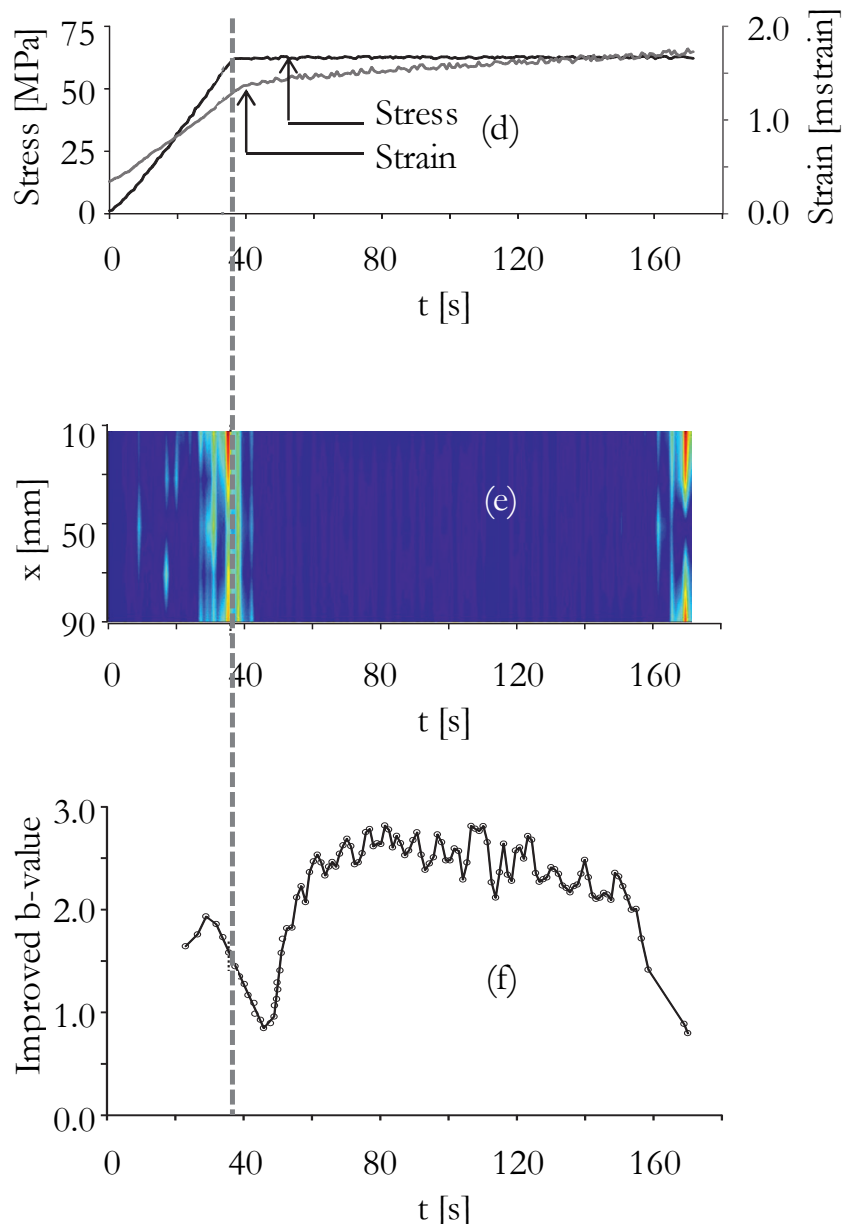

Figure 6: Time variation of the axial stress and the axial strain $(\mathrm{a}, \mathrm{d})$, the spatial distribution of the PSC energy (b, e), and the I $b$-value (c, f), for the first (left column) and the second (right column) loading loop. 
specimen (almost $10 \mathrm{~s}$ earlier), the electrodes closer to the specimen's upper and lower bases start suddenly recording PSC signals of high energy. This observation is in accordance with what was concluded from Figs.4(h-l). During this last time interval only minor emissions are recorded from the area around the specimen's mid-height. This sudden electric activity, which starts almost $10 \mathrm{~s}$ before the specimen's collapse could be considered as an interesting pre-failure indicator, especially due to the time distance from the specimen's collapse.

Concerning the amplitudes of the AE events, they were processed in the direction of conducting b-value analysis for both loading loops. In this context, the improved b-value $(\mathrm{I} b)$ was calculated for each one of the two loading loops according to well-known formalisms $[5,19,40,41]$. The time variation of the $\mathrm{I} b$-value calculated in this way during the two loading loops is plotted in Figs.6(c,f). Concerning the first loading loop, the $\mathrm{I} b$-value (after a slight increase) decreases with increasing axial stress. This decrease is abruptly terminated when the stress attains its maximum value and an abrupt increase is observed. From this point on, the $\mathrm{I} b$-value remains practically constant at a relatively high level (equal to about 2.0), implying that no strong AE events are recorded. During the second loading loop, the $\mathrm{I} b$-values exhibit a similar behaviour. The most important observation during the second loading loop is that about $70 \mathrm{~s}$ after the stress has reached its maximum value (and is kept constant) the $\mathrm{I} b$-value starts decreasing gradually implying that new AE events of higher amplitudes occur. Then a characteristic drop of the I $b$-value at levels approaching $\mathrm{I} b=1$ is observed, slightly before the specimen's collapse, in excellent accordance with the respective behaviour of the PSC energy.

\section{CONCLUDING REMARKS}

7 he Pressure Stimulated Currents (PSC) and the Acoustic Emission (AE) experimental techniques were employed according to a combined manner, in order to study the mechanical response of marble specimens subjected to successive compressive loading-unloading-reloading loops at stress levels very closely approaching the compressive strength of the specific marble variety. The two techniques were here combined for the first time (to the authors' best knowledge) in order to assess the spatiotemporal damage evolution and at the same time to explore the applicability of their data as estimators of proximity to fracture.

In this direction, the PSC technique was here developed further by using a grid of electrode pairs, aiming to the collection of PSC emissions from multiple sensors. It was clearly shown that the updated PSC technique provides valuable information regarding the location of the PSC source and the spatiotemporal evolution of damage within the specimens.

The correlation of the two techniques was here implemented in terms of the PSC energy and the improved b-value (I $b)$ of the AE events. The comparison indicated very good qualitative and quantitative agreement between the results obtained by the two techniques.

Moreover, it was proven that both the as above mentioned quantities (PSC energy and I $b$-value) provide clear indicators of the proximity to fracture: The PSC energy starts increasing abruptly well before the specimens' collapse while at the same time the $\mathrm{I} b$-value exhibits a characteristic steep drop towards the $\mathrm{I} b=1$ critical limit.

\section{REFERENCES}

[1] Chu, T.C., Ranson, W.F. and Sutton, M.A. (1985). Applications of digital-image-correlation techniques to experimental mechanics, Exp. Mech., 25(3), pp. 232-244.

[2] Iliopoulos, A. and Michopoulos, J.G. (2012). Direct strain imaging for full field measurements, ASME 2012 International Design Engineering Technical Conferences \& Computers and Information in Engineering Conference IDETC/ CIE, August 13-15, 2012, Chicago, IL, USA.

[3] Lavrov, A. (2005). Fracture-induced physical phenomena and memory effects in rocks: a review, Strain, 41, pp. 135149.

[4] Lockner, D. (1993). The role of acoustic emission in the study of rock fracture, Int. J. Rock Mech. Min. Sci. Geomech. Abstr., 3, pp. 883-899.

[5] Rao, M.V.M.S. and Lakschmi, P.K.J. (2005). Analysis of b-value and improved b-value of acoustic emissions accompanying rock fracture, Curr. Sci. India, 89, pp. 1577-1582.

[6] Stanchits, S., Dresen, G. and Vinciguerra, S. (2006). Ultrasonic velocities, acoustic emission characteristics and crack damage of basalt and granite, Pure Appl. Geophys, 163(5-6), pp. 975-994.

[7] Yamada, I., Masuda, K. and Mizutani, H. (1989). Electromagnetic and acoustic emission associated with rock fracture, Phys. Earth. Planet. In., 57, pp. 157-168. 
[8] Stavrakas, I., Triantis, D., Agioutantis, Z., Maurigiannakis, S., Saltas, V., Vallianatos, F. and Clarke, M. (2004). Pressure stimulated currents in rocks and their correlation with mechanical properties, Nat. Hazards Earth Sys., 4, pp. 563-567.

[9] Vallianatos, F., Triantis, D., Tzanis, A., Anastasiadis, C. and Stavrakas, I. (2004). Electric earthquake precursors: From laboratory results to field observations, Phys. Chem. Earth, 29, pp. 339-351.

[10] Stavrakas, I., Anastasiadis, C., Triantis, D. and Vallianatos, F. (2003). Piezo Stimulated currents in marble samples: Precursory and concurrent - with - failure signals, Nat. Hazards Earth Sys., 3, pp. 243-247.

[11] Triantis, D., Anastasiadis, C., Vallianatos, F., Kyriazis, P. and Nover, G. (2007) Electric signal emissions during repeated abrupt uniaxial compressional stress steps in amphibolite from KTB drilling, Nat. Hazards Earth Sys., 7, pp. 149-154.

[12] Kyriazopoulos, A., Anastasiadis, C., Triantis, D. and Brown, J.C. (2011). Non-destructive evaluation of cement-based materials from pressure stimulated electrical emission: Preliminary results, Const. Build. Mater., 25, pp. 1980-1990.

[13] Stergiopoulos, C., Stavrakas, I., Triantis, D., Vallianatos, F. and Stonham, J. (2015). Predicting fracture of mortar beams under three-point bending using non-extensive statistical modelling of electric emissions, Physica A, 419, pp. 603-611.

[14] Cartwright-Taylor, A., Vallianatos, F. and Sammonds, P. (2014). Superstatistical view of stress-induced electric current fluctuations in rocks, Physica A, 414, pp. 368-377.

[15] Zhonghui, L., Wang, E. and He, M. (2015). Laboratory studies of electric current generated during fracture of coal and rock in rock burst coal mine, J. Mining, 2015: 235636.

[16] Sun, M., Liu, Q., Li, Z. and Wang, E. (2002). Electrical emission in mortar under low compressive loading, Cement Concrete Res., 32, pp. 47-50.

[17] Archer, J.W., Dobbs, M.R., Aydin, A., Reeves, H.J. and Prance, R.J. (2016). Measurement and correlation of acoustic emissions and pressure stimulated voltages in rock using an electric potential sensor, Int. J. Rock Mech. \& Mining Sci., 89, pp. 26-33.

[18] Dann, D., Demikhova, P., Fursa, T. and Kuimova, M. (2014). Research of electrical response communication parameters on the pulse mechanical impact with the stress-strain state of concrete under uniaxial compression, IOP Conf. Series: Materials Science and Engineering, 66: 012036.

[19] Shiotani, T., Fujii, K., Aoki, T. and Amou, K. (1994). Evaluation of progressive failure using AE sources and improved b-value on slope model tests, Prog. Acoust. Emission, VII, pp. 529-534.

[20] Tassogiannopoulos, A.G. (1986). A contribution to the study of the properties of structural natural stones of Greece (in Greek), National Technical University of Athens, Doctoral Dissertation, Athens, Greece.

[21] Exadaktylos, G.E., Vardoulakis, I. and Kourkoulis, S.K. (2001) Influence of nonlinearity and double elasticity on flexure of rock beams - II. Characterization of Dionysos marble, Int. J. Solids Struct., 38, pp. 4119-4145.

[22] Theocaris, P.S. and Coroneos, E. (1979). Experimental study of the stability of Parthenon (in Greek). Publications of the Academy of Athens, 44, pp. 1-80.

[23] Zambas, C. (2004). Mechanical properties of Pentelik marble [in Greek], Ministry of Culture, Committee for the Preservation of Parthenon Publications, Athens, Greece.

[24] Vardoulakis, I. and Kourkoulis, S.K. (1997). Mechanical properties of Dionysos marble, Final report of the European Union Environment project EV5V-CT93-0300 "Monuments under seismic action", Nat. Tech. Univ. Athens, Athens.

[25] Labuz, J.F., Bridell J.M. (1993). Reducing frictional constraint in compression testing through lubrication, Int. J. Rock Mech. Min. Sci. \& Geomech. Abstr., 30, pp. 451-455.

[26] Drescher, A. and Vardoulakis, I. (1982). Geometric softening in triaxial test on granular materials, Geotechnique, 32, pp. 291-303.

[27] Read, H.E. and Hegemier, G.A. (1984). Strain softening of rock, soil and concrete - A review article, Mech. Mater., 3(4), pp. 271-294.

[28] Van Vliet, M.R.A. and van Mier, J.G.M. (1995). Concrete under uniaxial compression, Report 25.5-95-9, TU Delft, Faculty of Civil Engineering, The Netherlands.

[29] Kourkoulis, S.K. (2012). Critical aspects of the mechanical behaviour and failure of Dionysos marble under direct tension, J. Serbian Soc. Comp. Mech., 6(1), pp. 199-215.

[30] Kourkoulis, S.K., Exadaktylos, G. and Vardoulakis, I. (2000). The degradation of the mechanical properties of natural building stones: An "elastic-damage" model. In: Structural Integrity in the $21^{\text {st }}$ Century. The lifetime of plant, structures and components: evaluation, design, extension and management, J.H. Edwards et al. (eds.), EMAS Publishing, Solihull, UK, pp.171-179.

[31] Vardoulakis, I., Labuz, J., Papamichos, E. and Tronvoll, J. (1998). Continuum fracture mechanics of uniaxial compression on brittle materials, Int. J. Solids Struct., 35, pp. 4313-4335.

[32] Van Vliet, M.R.A. and van Mier, J.G.M. (1996). Experimental investigation of concrete fracture under uniaxial compression, Mech. Cohes-Frict. Mat., 1(1), pp. 115-127. 
[33] Triantis, D., Anastasiadis, C. and Stavrakas, I. (2008). The correlation of electrical charge with strain on stressed rock samples, Nat. Hazards Earth Sys., 8, pp. 1243-1248.

[34] Alexandridis, A., Triantis, D., Stavrakas, I. and Stergiopoulos, C. (2012). A neural network approach for compressive strength prediction in cement-based materials through the study of pressure-stimulated electrical signals, Const. Build. Mater., 30, pp. 294-300.

[35] Triantis, D., Vallianatos, F., Stavrakas, I. and Hloupis, G. (2012). Relaxation phenomena of electrical signal emissions from rock following application of abrupt mechanical stress, Ann Geophys-Italy, 55(1), pp. $207-212$.

[36] Lavrov, A. (2003.) The Kaiser effect in rocks: principles and stress estimation techniques, Int. J. Rock Mech. Min. Sci., 40(2), pp. 151-171.

[37] Li, C. and Nordlund, E. (1993). Assessment of damage in rock using the Kaiser effect of acoustic emission, Int. J. Rock Mech. Min. Sci. \& Geomech. Abstr., 30(7), pp. 943-946.

[38] Tsimogiannis, A., Georgali, B. and Anastassopoulos, A. (2000). Acoustic Emission/Acousto-ultrasonic data fusion for damage evaluation in concrete, J. Acoustic Emissions, 18, pp. 21-28.

[39] Weber, Z., Svadbik, P., Korenska, M. and Pazdera, L. (2000). Concrete crossbeam diagnosis by acoustic emission method, J. Acoustic Emissions, 18, pp. 29-32.

[40] Colombo, S., Main, I.G. and Forde, M.C. (2003). Assessing damage of reinforced concrete beam using "b-value" analysis of acoustic emission signals, J. Mat. Civil Eng., 15, pp. 280-286.

[41] Rao, M.V.M.S. (1997). Acoustic emission signatures of rocks and concrete stressed to failure in compression under laboratory conditions, In: Proc. $4^{\text {th }}$ National Workshop on Acoustic Emission (NAWACE- 97), Mumbai, August 1997, pp. 31-41. 